\title{
Bajas dosis de aspirina previenen el tromboembolismo venoso en pacientes de alto riego quirúrgico
}

Prevention of pulmonary embolism and deep vein thrombosis with low dose aspirin: pulmonary embolism prevention (PEP) trial. Pulmonary embolism prevention (PEP) trial collaborative group. The Lancet 2000, 355: 1295-1302

\section{Objetivo}

Evaluar si la aspirina en bajas dosis disminuye el riesgo de tromboembolismo venoso en pacientes con condiciones medico-quirurgicas de alto riesgo.

\section{Diseño}

Estudio aleatorizado, multicéntrico, doble ciego, con un seguimiento de 35 días.

\section{Lugar}

170 hospitales de Australia, Nueva Zelanda, Sudáfrica, Suiza y Gran Bretaña.

\section{Pacientes}

Se incluyeron 13356 pacientes a quienes se le realizaría cirugía por fractura de cadera y 4088 pacientes a quienes se les realizaría artroplastia electiva de rodilla o cadera. La edad promedio fue de 79 años y el $79 \%$ eran mujeres.

\section{Intervención}

Se aletorizó a los pacientes a recibir $160 \mathrm{mg} /$ día de aspirina o placebo, comenzando en forma pre-operatoria y continuando por 35 días. Los pacientes recibieron otras tromboprofilaxis según necesidad.

\section{Medición de los resultados principales}

Los resultados principales se definieron como mortalidad y morbilidad intrahospitalaria hasta el día 35. El seguimiento para los eventos no fatales (trombosis venosa profunda, embolismo pulmonar, infarto de miocardio, accidente cerebrovascular y episodios de sangrado) continuó durante la permanencia en el hospital, mientras que el seguimiento para las muertes se realizó durante la estadia y posteriormente a la misma.

\section{Resultados principales}

Entre los pacientes con fractura de cadera, aquellos que recibieron aspirina tuvieron un $43 \%$ menos de riesgo de desarrollar embolia pulmonar (IC95\%: 18 a 60) y una reducción del riesgo de trombosis venosa profunda sintomática de 29\% (IC95\%: 3 a 48). La embolia de pulmón y la trombosis venosa profunda fueron confirmadas en $105(1,6 \%)$ de los 6679 pacientes que recibieron aspirina lo que, comparado con los 165 eventos (2,5\%) de los 6677 que recibieron placebo. Esto representa una reducción global absoluta de nueve eventos por cada mil pacientes tratados.

La aspirina previno 4 embolias pulmonares fatales por cada mil pacientes tratados (18 muertes del grupo de aspirina vs 43 del grupo placebo) lo que representa una reducción relativa del 58\% (IC95\%: 27 a $76 ; p=0,002)$. No se encontraron diferencias significativas en las muertes por otras causas vasculares o no vasculares.

Las muertes por sangrado fueron pocas (13 en los tratados con aspirina y 15 en quienes recibieron placebo). Sin embargo, hubo diferencias significativas en la necesidad de transfusiones posoperatorias (seis más cada mil pacientes en el grupo aspirina; $p=0,04$ ). Entre los pacientes con artroplastia electiva los porcentajes de tromboembolismo venoso fueron menores, pero los efectos proporcionales de la aspirina fueron compatibles con los de los pacientes con fractura de cadera.

\section{Conclusiones}

Estos resultados, junto con los de metaanálisis previos, muestran que la aspirina reduce el riesgo de embolismo pulmonar y trombosis venosa profunda en al menos un tercio durante el período de mayor riesgo. Por lo tanto existe buena evidencia para recomendar la aspirina en forma rutinaria en grupos con condiciones medico-quirúrgicas que impliquen alto riesgo de tromboembolismo.

\section{COMENTARIO}

Este estudio fue realizado con el fin de confirmar o refutar los resultados que metaanálisis de estudios previos ${ }^{1}$ habían sugerido respecto a que el tratamiento con bajas dosis de aspirina es efectivo en la prevención de tromboembolismo venoso en pacientes de alto riesgo.

Si bien los resultados muestran con fuerte evidencia que la aspirina disminuye la frecuencia de tromboembolismo venoso sintomático luego de una cirugía ortopédica mayor, sería necesario considerar que el objetivo de este estudio no fue comparar la aspirina con otras profilaxis farmacológicas como la heparina de bajo peso molecular. Este punto es importante ya que diversos ensayos clinicos ${ }^{2-5}$ han demostrado la eficacia de la heparina en la profilaxis de tromboembolismo venoso luego de cirugías de alto riesgo ( $3 \%$ de redución del riesgo absoluto de TVP y $1,6 \%$ de embolia pulmonar).

Aunque no se ha determinado la duración ideal del tratamiento, el conocimiento de la existencia de TVP asintomática que produce TEP ha
Llevado a extender la profilaxis hasta un mes después del alta hospitaLaria. El presente estudio no evalúa el desempeño comparativo entre la aspirina y la heparina de bajo peso molecular en pacientes sometidos a cirugías de alto riesgo. Tampoco queda claro si debe reemplazar o ser administrada junto con otras medidas farmacológicas en el post-operatorio inmediato. Aún más, aunque no haya sido estadísticamente significativo, pareciera que el beneficio de la aspirina como profilaxis fue mayor luego de la primera semana, momento en el cual los pacientes ya habían sido dados de alta y no estaban recibiendo otra forma de profilaxis.

Podría considerarse la utilidad de la aspirina en aquellos pacientes con contraindicaciones para recibir heparina, aunque sería necesario una mayor investigación para determinar la mejor estrategia de manejo y, en especial, para establecer la mejor profilaxis luego del alta hospitalaria, teniendo en cuenta además las características de cada paciente en particular.

\section{Dra. María Gabriela Fabbro}

Servicio de Clínica Médica. Hospital Italiano de Buenos Aires.

\section{Referencias}

1-Collins R, Scrimgeour A, Yusuf S, Peto R. Reduction in fatal pulmonary embolism and venous thrombosis by perioperative administration of subcutaneous heparin: overview of results of randomized trials in general, orthopedic and urologycal surgery. N. Engl J Med 1988; 318: 1162-73

2- Nurmohamed MT, Rosendaal FR, Büller HR, et al. Low molecular weight heparin versus standard heparin in general and orthopedic surgery: a metaanalyses. Lancet 1992; $340: 152-56$ 3- Koch A, Bouges S, Ziegler S, Dinkel H, Daures JP, Victor N. Low molecular weight heparin and unfractionated heparin in thrombosis prophylaxis after major surgical intervention: update of previousmeta-analyses. Br J Surg 1997; 84: 750-59

4- Antiplatelet trialists' Collaboration. Collaborative overview of randomized trials of antiplatelet therapy- III : reduction in venous thrombosis and pulmonary embolism by antiplatelet prophylaxis among surgical and medical patients. BMJ 1994; 309: 1215-17

5- MacMahon S, RodgersA, Collins R, Farrel B. Antiplatelet therapy to prevent thrombosis after hip fracture. J Bone Joint Surg Br 1994; 76-B: 521-24. 